\title{
Systemic Treatment for Advanced Hepatocellular Carcinoma
}

\author{
Mohamed Bouattour $^{\mathrm{a}}$ Neil Mehta ${ }^{\mathrm{b}}$ Aiwu R. He $\mathrm{H}^{\mathrm{c}}$ Emil I. Cohen ${ }^{\mathrm{d}}$ \\ Jean-Charles Nault ${ }^{e}$ f \\ aDepartment of Digestive Oncology, Hôpital Beaujon, APHP Hôpitaux Universitaires \\ Paris Nord Val de Seine, Clichy, France; bUCSF Medical Center, San Francisco, CA, USA; \\ 'Department of Medical Oncology, MedStar Georgetown University Hospital, Washington, \\ DC, USA; d Department of Radiology, MedStar Georgetown University Hospital, Washington, \\ DC, USA; ' Liver Unit, Hôpital Jean Verdier, APHP Hôpitaux Universitaires Paris Seine-Saint-Denis, \\ Paris, France; ${ }^{f}$ Centre de Recherche des Cordeliers, Sorbonne Université, Inserm, USPC, \\ Université Paris Descartes, Université Paris Diderot, Université Paris 13, Functional Genomics of \\ Solid Tumors Laboratory, Paris, France
}

\author{
Keywords \\ Hepatocellular carcinoma - Patient characteristics - Precision medicine - Targeted therapy · \\ Treatment sequence
}

\begin{abstract}
Background: Patients with advanced hepatocellular carcinoma (HCC) have a poor prognosis. First-line sorafenib has been the standard of care for a decade, but the treatment landscape is expanding. This review provides a practical overview of current and future systemic treatment options for advanced HCC and their place in clinical practice. Summary: First-line sorafenib and lenvatinib have shown to improve the survival of patients with advanced HCC. In the second line, regorafenib provides benefit for patients who previously tolerated sorafenib. Anti-PD1 antibodies, nivolumab and pembrolizumab, recently became available for secondline use in the US. Ramucirumab (for patients with $\alpha$-fetoprotein [AFP] levels $\geq 400$ ) and cabozantinib present potential future second-line treatment options. Combinations of systemic and locoregional treatment, such as radiofrequency ablation or selective internal radiotherapy, require further research. Precision medicine has not yet been translated into clinical practice, as the most common driver mutations (TERT promoter, CTNNB1, TP53, and ARID1A mutations) have not yet been shown to be suitable therapeutic targets. However, our growing understanding of signaling pathways and efforts in drug development are expected to pave the way for precision medicine in HCC in the future. Evaluating the place for the current and novel systemic treatment options in clinical practice can be challenging due to the diverse toxicity profiles of the treatment options and characteristics of the patient population.
\end{abstract}


Bouattour et al.: Systemic Treatment for Advanced HCC

Sorafenib data elucidate the effect patient characteristics (such as the performance score, Child-Pugh class, AFP, etiology of the underlying disease, and level of macrovascular invasion and extrahepatic spread) may have on outcomes in advanced stages. Key Messages: Lenvatinib is expected to join sorafenib as a preferred first-line treatment in advanced HCC. In the second line, the treatment of choice, regorafenib, is soon expected to be accompanied by cabozantinib and ramucirumab in patients with AFP $\geq 400 \mathrm{ng} / \mathrm{mL}$, whereas nivolumab and pembrolizumab present second-line alternatives in the US.

(C) 2019 S. Karger AG, Base

\section{Introduction}

Hepatocellular carcinoma (HCC) is the second most common cause of cancer-related death [1]. HCC is almost invariably associated with underlying risk factors, such as chronic infections with hepatitis B (HBV) or C (HCV), metabolic syndrome, and alcohol abuse [2]. Many patients present with advanced-stage disease defined as Barcelona Clinic Liver Cancer (BCLC) stage C: cancer-related symptoms (performance status 1-2), preserved liver function, and portal invasion or extrahepatic spread (EHS) [2,3]. These patients have a poor prognosis. Without treatment, the median overall survival (OS) of patients included in the SHARP trial (mixing BCLC B progressing after TACE and BCLC C patients) is around 8 months [4]. In the SHARP trial, the subgroup of BCLC C patients in the placebo arm had a median OS of 7 months, whereas BCLC B patients had a median OS of 11.4 months [5].

Sorafenib, a multi-kinase inhibitor with anti-proliferative and anti-angiogenic effects, has been the global standard of care for advanced HCC for a decade. First-line sorafenib improved OS in two large, phase 3, randomized controlled trials (RCTs) [4, 6]. In 2017, regorafenib was registered as second-line treatment in the US and Europe, whereas nivolumab and pembrolizumab were registered for second-line use only in the US [2, 7-9]. This review outlines the current data on common questions regarding the systemic treatment of advanced HCC and their practical implications, aiming to assist physicians in making treatment decisions for these patients.

\section{Questions and Answers on the Systemic Treatment for Advanced HCC}

\section{What Is the Impact of Patient Characteristics on Outcomes with Sorafenib?}

The main prognostic and predictive studies in advanced HCC have been performed in sorafenib-treated patients. In the general SHARP trial population, first-line sorafenib improved the median OS by nearly 3 months compared with placebo (10.7 vs. 7.9 months; hazard ratio $[\mathrm{HR}] 0.69 ; p<0.001$ ). The median time to radiological progression (TTP) improved from 5.5 to 2.8 months $(p<0.001)$ [4]. In the Asia-Pacific (AP) trial, sorafenib was evaluated in China, South Korea, and Taiwan [6]. The survival benefit of sorafenib versus placebo was similar to the SHARP trial (median OS 6.5 vs. 4.2 months; HR 0.68; $p=0.014$ ), with a median TTP of 2.8 versus 1.4 months ( $p=0.0005)$. Common sorafenib-associated adverse events (AEs) included diarrhea (39/26\%; 8/6\% grade 3/4), hand-foot-skin reaction (HFSR) $(21 / 45 \%$; 8/11\% grade $3 / 4)$, and fatigue $(22 / 20 \% ; 4 / 3 \%$ grade $3 / 4)$, in the SHARP/AP trials, respectively $[4,6]$.

The AP and SHARP trials illustrated the impact of patient characteristics on outcomes in advanced stages of HCC $[4,6]$. Although the inclusion criteria were similar, there were differences in the etiology of the patient populations. For example, the SHARP trial included patients 


\section{Liver
Cancer}

\begin{tabular}{|c|c|}
\hline \multicolumn{2}{|c|}{-358} \\
\hline DOI: 10.1159/000496439 & $\begin{array}{l}\text { (c) } 2019 \text { S. Karger AG, Basel } \\
\text { www.karger.com/lic }\end{array}$ \\
\hline
\end{tabular}

Bouattour et al.: Systemic Treatment for Advanced HCC

from Europe, North America, South America, and Australasia in whom the predominant etiologies were HCV and alcohol abuse, whereas the AP study included an Asian population with HBV as the main etiology. Furthermore, the Asian patients had more advanced disease (EHS, performance score, and number of tumors) than the SHARP population. The absolute OS and TTP were lower in the AP than in the SHARP trial. However, the HRs for survival benefit were similar.

(1a) What Is the Impact of Liver Function according to the Child-Pugh Class on Treatment Efficacy?

The majority of patients with HCC have concurrent cirrhosis. The two sorafenib studies only included patients with good liver function (Child-Pugh A), as sorafenib may be deleterious in case of decompensated cirrhosis. Cohort studies have shown that Child-Pugh B patients have a lower OS benefit from sorafenib than Child-Pugh A patients [10,11]. A phase 2 trial showed more severe liver toxicities (including hyperbilirubinemia, ascites, and encephalopathy) in Child-Pugh B versus Child-Pugh A patients, despite similar pharmacokinetic and toxicity profiles [12]. Sorafenib should be used with caution in Child-Pugh B patients, as there is a narrow margin between an unknown clinical benefit and the risk of toxicities and liver decompensation [13]. In the GIDEON study, some Child-Pugh B and C patients have been treated with sorafenib without obvious deleterious effects on liver function. However, this study was not designed to assess this issue [14]. Consequently, sorafenib is still contraindicated in Child-Pugh C patients, because of the limited life expectancy and the low magnitude of benefit in this population.

(1b) What Is the Impact of Macrovascular Invasion and EHS on Treatment Efficacy?

A large proportion of patients with advanced HCC have macrovascular invasion (MVI) and/or EHS, which impacts OS $[4,6]$. In the SHARP trial, the median OS was 8.9 months in patients with MVI and/or EHS versus 14.5 months in those without [5]. A combined analysis of the SHARP and AP trials confirmed that patients with EHS have a smaller absolute OS benefit from sorafenib (HR $=0.84$ with EHS vs. 0.55 without EHS) [15]. More data are needed about the prognostic value of the extent of MVI and the numbers, size, and location of EHS in patients treated with tyrosine kinase inhibitors (TKIs).

(1c) Does the Etiology of the Underlying Disease Impact Treatment Efficacy?

In the SHARP trial, the most frequently reported factors underlying HCC were HCV and alcohol abuse (29 and 26\%) [4]. Only 19\% of patients were infected with HBV (19\%), the major underlying disease in the AP trial $(71 \%)[4,6]$. HBV-positive patients had a lower median OS in the AP trial compared to patients included in the SHARP trial. However, it is not obvious that this difference is strictly due to the HBV infection or more probably related to the difference in baseline characteristics of the patients and tumors [15].

Some data suggest that patients with HBV-related HCC have poorer prognosis and OS than those infected with HCV [16]. A meta-analysis of three RCTs suggested that the effect of sorafenib was not significant in patients with HBV [17]. The efficacy in patients with HCV-related HCC was confirmed by a subset analysis from the SHARP trial, showing an OS of 14.0 months with sorafenib versus 7.4 months with placebo (HR 0.50) [5]. However, the AP trial also showed a significantly increased OS for sorafenib versus placebo (HR 0.74), while more than $70 \%$ of patients were HBV positive $[6,15]$. Cross-trial comparisons should be interpreted with caution. Interestingly, the phase 3 REFLECT trial has shown somewhat more survival benefit from lenvatinib versus sorafenib in patients with HBV (13.4 vs. 10.2 months; HR 0.83) [18]. However, this study was designed as a noninferiority trial and superiority over sorafenib was not reached. Overall, treatment choice cannot be solely based on the etiology of the underlying liver disease, as these data are derived from post hoc subgroup analysis of RCTs. 
(1d) What Is the Impact of $\alpha$-Fetoprotein Levels on Treatment Efficacy?

A multivariate subgroup analysis of the SHARP and AP studies showed that $\alpha$-fetoprotein (AFP) levels $>200 \mathrm{ng} / \mathrm{mL}$ were a strong prognostic factor of poor OS with sorafenib [15]. In the REFLECT study, patients with baseline AFP levels $<200 \mathrm{ng} / \mathrm{mL}$ had a longer OS than those with AFP levels $\geq 200 \mathrm{ng} / \mathrm{mL}$, both in the lenvatinib and sorafenib group, confirming a prognostic value of this biomarker in each treatment arm [18]. Conversely, a post hoc subgroup analysis showed that ramucirumab significantly increased OS in patients with high AFP levels (7.8 vs. 4.2 months, $p=0.006$ ) [19]. The phase 3 REACH-2 trial has compared second-line ramucirumab with placebo in patients with a serum AFP $\geq 400 \mathrm{ng} / \mathrm{mL}$ (NCT02435433). Presentation of results at the ASCO meeting showed an increased OS in the ramucirumab trial, suggesting that the AFP level is a predictive marker of response to ramucirumab [20].

(2) What Are the Approved Second-Line Treatment Options for Advanced HCC?

A phase 2 RCT showed no benefit of dose escalation of sorafenib in progressive patients [21]. In the last decade, several phase 3 RCTs testing novel, mainly targeted therapies in advanced HCC, failed to show improved OS due to toxicity or, more frequently, absence of efficacy (Table 1) [4, 6, 19, 20, 22-32]. However, regorafenib (FDA and EMA) and nivolumab in 2017 and pembrolizumab in 2018 were approved (by the FDA and EMA and by the FDA alone, respectively) for second-line use in patients previously treated with sorafenib.

(2a) What Is the Role of Regorafenib in the Second-Line Treatment of Advanced HCC?

In December 2016, regorafenib ended the succession of negative results in advanced HCC $[8,33]$. Regorafenib is an anti-angiogenic TKI that also targets stroma and oncogenic signaling, such as the mitogen-activated protein kinase pathway [8]. In the phase 3 RESORCE RCT, secondline regorafenib increased the OS by 2.8 months versus placebo (10.6 vs. 7.8 months; $p<0.0001$; HR 0.63 ) in patients with radiological progression on sorafenib. However, this study included a selected patient population, consisting of patients with Child-Pugh A cirrhosis and Eastern Cooperative Oncology Group performance status (ECOG-PS) scores $\leq 1$, who tolerated sorafenib $\geq 400 \mathrm{mg}$ /day for $\geq 20$ of the last 28 days of treatment. Interestingly, patients receiving secondline placebo in the RESORCE trial had the same OS (7.8 months) as patients receiving first-line placebo in the SHARP trial [33]. This illustrates the more indolent patient population included in second-line trials. Furthermore, experience with subgroups not included in the RESORCE study is limited. Sequential treatment with sorafenib followed by regorafenib led to a median OS of 26 months [34]. However, liver function impairment or poor performance status prevented some patients from benefitting from a second-line treatment.

The main AEs of regorafenib were similar to sorafenib and occurred frequently, with HFSR reported in 53\% (13\% grade 3), diarrhea in 41\% (3\% grade 3), fatigue in $40 \%$ (9\% grade 3 ), and hypertension in 31\% (15\% grade 3/4) of patients [8]. Grade 3/4 AEs occurred in $67 \%$ of patients under regorafenib versus $39 \%$ under placebo and called for dose reductions or interruptions in 54 and $10 \%$ of patients, respectively.

Regorafenib was approved by the FDA and EMA as second-line treatment after sorafenib failure in patients with advanced HCC and is currently the recommended second-line treatment in Child-Pugh A patients with an ECOG-PS score of 0-1 who tolerated sorafenib in the first line. Currently, patients with Child-Pugh B cirrhosis, significant comorbidities or sorafenib intolerance have been excluded from the RESORCE trial and should not be treated with regorafenib [35]. In these populations, efficacy and tolerance are still unknown. Careful evaluation of second-line regorafenib in the real-world setting is required; a noninterventional study is currently ongoing (NCT03289273). 


\section{Liver
Cancer}

\begin{tabular}{|c|c|}
\hline \multicolumn{2}{|c|}{-358} \\
\hline DOI: 10.1159/000496439 & $\begin{array}{l}\text { (c) } 2019 \text { S. Karger AG, Basel } \\
\text { www.karger.com/lic }\end{array}$ \\
\hline
\end{tabular}

Bouattour et al.: Systemic Treatment for Advanced HCC

(2b) What Is the Role of Nivolumab and Pembrolizumab in the Second-Line Treatment of Advanced HCC?

Immunotherapy aims to unleash the immune response against cancer cells. The monoclonal antibodies directed against programmed cell death protein 1 (PD1), nivolumab, and pembrolizumab were recently approved by the FDA for the second-line treatment of advanced HCC. The FDA approval of nivolumab in 2017 was based on a nonrandomized, open-label, phase 1/2 study (CheckMate-040), in which nivolumab showed a median OS of 15 months, an overall response rate (ORR) of 19\%, and a median duration of response of 9.9 months in the overall population [9]. The main AEs included pruritus (19\%), rash (16\%), and diarrhea (14\%). Autoimmune AEs were rare. In 2018, second-line pembrolizumab showed promising data from an ongoing phase 2 trial (Keynote-224), with an ORR of $17 \%$, a median progressionfree survival (PFS) of 4.9 months and a median OS of 12.9 months and was approved by the FDA [36].

Although data from these studies were encouraging enough for the FDA to approve nivolumab and pembrolizumab, the CheckMate-040 study was an early-stage study with a heterogeneous population, mixing sorafenib-naive and pretreated patients. Moreover, neither CheckMate-040 nor Keynote-224 included a control arm. Continued approval may depend on confirmation of the results and clinical benefit in the phase 3 RCTs expected for 2019.

What Will Be the Place of Novel Compounds in the Treatment of Advanced HCC?

Several novel systemic therapies are currently evaluated in RCTs, including targeted therapies and immunotherapy.

\section{Lenvatinib}

Lenvatinib is a multi-target kinase inhibitor directed against receptors for vascular endothelial growth factor (VEGF), fibroblast growth factors (FGF), platelet-derived growth factor, and the proto-oncogenes Kit and RET [37]. It was first evaluated in a Japanese phase 2 study. The majority of patients (59\%) were HCV-positive and 59\% had BCLC stage C HCC. Lenvatinib showed an ORR of 37\% and an OS of 18.7 months. Drug-related AEs seemed manageable and included arterial hypertension (76\%), HFSR (65\%), anorexia (61\%), and proteinuria (61\%). A total of 11 fatal AEs were reported, including hepatic failure and cerebral hemorrhage.

In February 2018, data from the phase 3, open-label, noninferiority RCT REFLECT, comparing first-line lenvatinib with sorafenib in patients with advanced HCC without MVI, were published [18]. Patients with main portal vein invasion, biliary invasion, and a tumor burden of more than $50 \%$ of the liver were excluded from this trial. Lenvatinib was noninferior to sorafenib (median OS 13.6 vs. 12.3 months; HR 0.92, 95\% confidence interval [CI] $0.79-1.06$ ) and was associated with a significantly higher PFS (7.4 vs. 3.7 months; $p<$ $0.00001)$, time to progression ( 8.9 vs. 3.7 months $p<0.0001)$ and ORR $(24.1 \%$ vs. $9.2 \% ; p<$ 0.00001 ) compared with sorafenib. Moreover, lenvatinib was associated with a higher ORR according to the modified Response Evaluation Criteria in Solid Tumors (mRECIST) criteria (from $24.1 \%$ in investigator review to $40.6 \%$ in masked independent review) than sorafenib (from $9.2 \%$ in investigator review to $12.4 \%$ in masked independent review). This was offset by an increased rate of treatment interruptions and discontinuations due to AEs with lenvatinib ( 40 vs. $32 \%$ and 9 vs. $7 \%$ ). The most common AEs in the lenvatinib group included hypertension (42\%), diarrhea (39\%), and decreased appetite (34\%). The OS of lenvatinib seemed to be consistent across subgroup analyses, including Western versus Eastern patients and patients with HBV versus HCV. Lenvatinib seemed to be more effective than sorafenib in the subgroup of HBV-infected patients. However, no definitive conclusions can be drawn from a subgroup analysis of an RCT. 


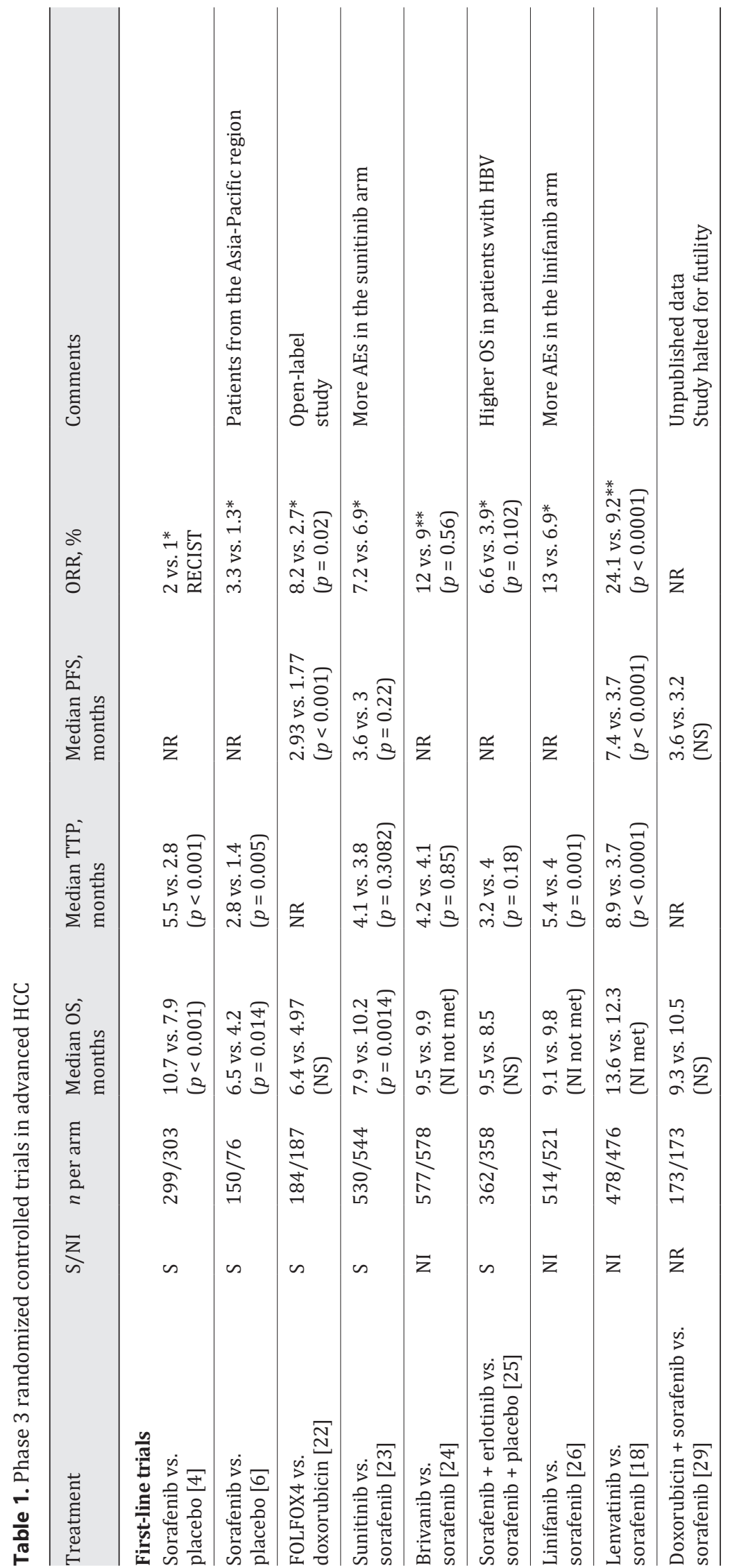




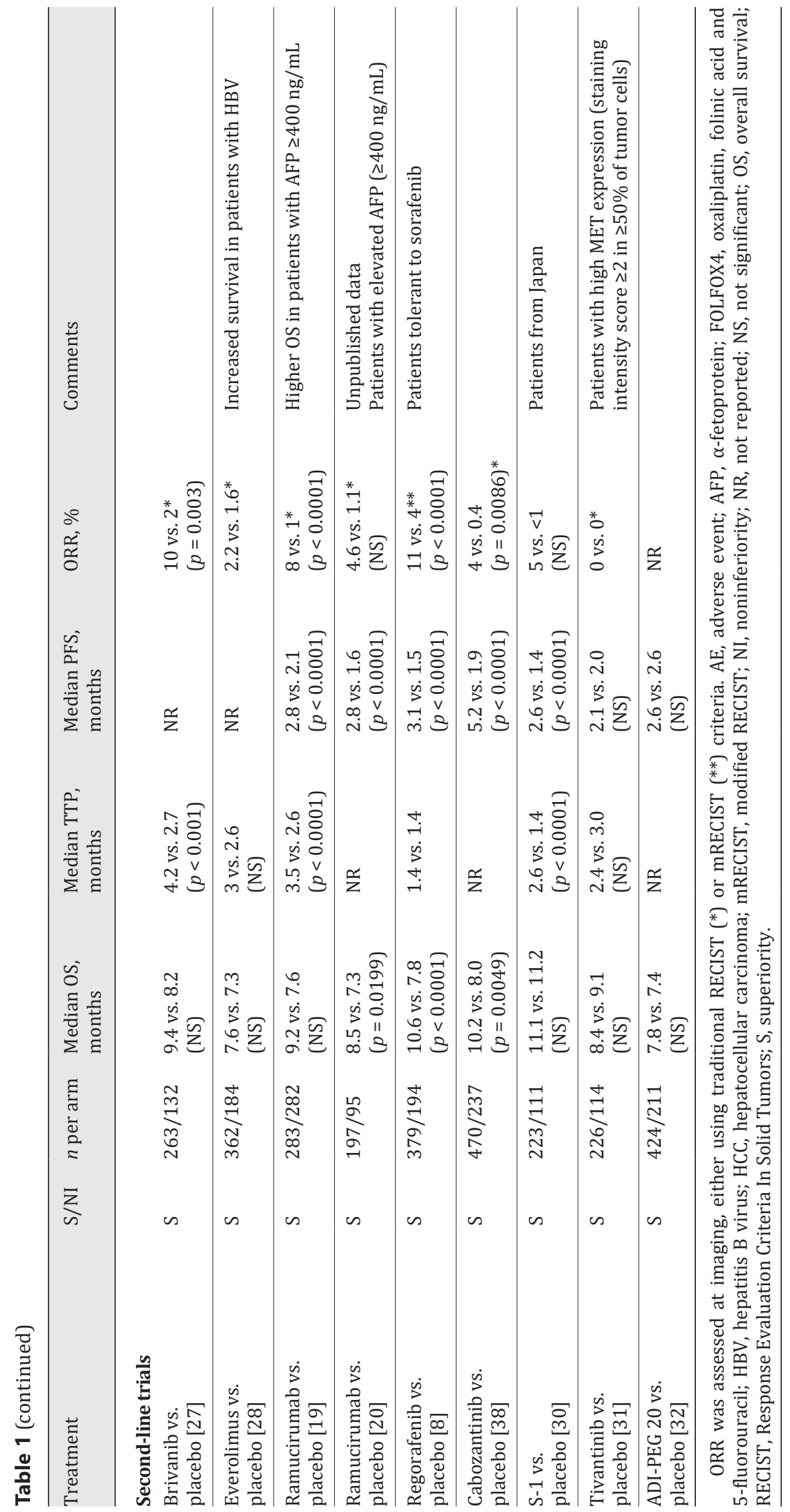




\section{Liver Cancer}

\begin{tabular}{|c|c|}
\hline \multicolumn{2}{|l|}{ Liver Cancer 2019;8:341-358 } \\
\hline DOI: 10.1159/000496439 & $\begin{array}{l}\text { (c) } 2019 \text { S. Karger AG, Basel } \\
\text { www.karger.com/lic }\end{array}$ \\
\hline
\end{tabular}

Bouattour et al.: Systemic Treatment for Advanced HCC

In 2018, lenvatinib was approved in the US and Japan. Further analysis of the phase 3 RCT data will be needed to elucidate the potential of lenvatinib as an alternative for sorafenib and to decide how to choose between lenvatinib and sorafenib. However, as the OS of lenvatinib is similar to sorafenib and no tumor biology-based biomarkers are available to guide treatment, the discussion on lenvatinib versus sorafenib may focus on toxicity and drug costs.

Cabozantinib

Cabozantinib is a multi-kinase inhibitor, targeting MET, AXL, and VEGF receptors, that was associated with a median OS of 11.5 months and an ORR of 5\% in a phase 2 study [38]. The main AEs were diarrhea (63\%), HFSR (56\%), and thrombocytopenia (37\%). The phase 3 CELESTIAL RCT showed an OS of 10.2 versus 8.0 months for cabozantinib versus placebo (HR 0.76; $p=0.005$ ) in the second or third line [39]. PFS was 5.2 versus 1.9 months (HR 0.44; $p<0.0001$ ). Although in favor of cabozantinib, the ORR was relatively limited in both groups ( 4 vs. $0.4 \% ; p<0.001$ ). However, cabozantinib almost doubled the disease control rate compared with placebo (partial response or stable disease; 64 vs. 33\%). The main grade $3 / 4$ AEs were HFSR (17\%) and hypertension (16\%). Cabozantinib is expected to be approved for second- or third-line use in 2018.

\section{Immunotherapy}

Cancer uses multiple mechanisms to evade the host immune response. Many novel compounds targeting different immune evasion mechanisms are being tested, as well as regimens combining immunotherapy with chemotherapy, targeted therapy, radiation, or another immunotherapy. These ongoing studies will bring more clarity on the role of immunotherapy in the treatment of HCC.

Phase 3 studies in patients with advanced HCC without major portal vein invasion comparing nivolumab to sorafenib in the first line and comparing pembrolizumab to placebo in the second line are ongoing (NCT02576509, NCT02702401). Tremelimumab, a blocker of cytotoxic T-lymphocyte-associated protein 4 (CTLA4), showed a partial response rate of $18 \%$ and a TTP of 6.5 months in a phase 2 study in HCV-related HCC [40]. A phase 3 RCT comparing durvalumab (anti-PDL1) plus tremelimumab (anti-CTLA4) versus sorafenib in patients with advanced HCC is currently underway (NCT032988451). In a phase 1 study in patients with advanced HCC, the combination of atezolizumab and VEGF inhibitor bevacizumab was safe and well tolerated, with promising early efficacy; the confirmed ORR per RECIST v1.1 was $61 \%$ by investigator assessment and $65 \%$ by independent review facility assessment (NCT02715531) [41]. Responses were observed in all subgroups, including patients with different etiologies, geographic regions, and baseline AFP levels. Responses were durable, with 10 responses ongoing for $\geq 6$ months and 3 of these ongoing for $\geq 1$ year per investigator assessment (median follow-up: 10.3 months). Median OS, PFS, and duration of response had not been reached at the clinical cutoff date (NCT02715531). The phase 3 RCT IMbrave150, comparing atezolizumab plus bevacizumab with sorafenib in patients with locally advanced or metastatic HCC, is ongoing (NCT03434379) [42]. The combination of the PD1 inhibitor pembrolizumab and the VEGFR/FGFR inhibitor lenvatinib is currently tested in a 2-part, phase $1 \mathrm{~b}$ trial (NCT03006926) [43]. It induced a confirmed ORR of $27 \%$ (95\% CI 12-48) in patients with unresectable HCC; when investigators included unconfirmed responses, the ORR was $42 \%$ (95\% CI 23-63).

Ongoing Phase 3 Trials with Targeted Therapies

Two other targeted drugs are currently being investigated in phase 3 trials in advanced HCC: ramucirumab and apatinib. Second-line ramucirumab, a monoclonal antibody targeting the VEGF receptor 2, failed to increase OS compared with placebo in the phase 3 REACH study 


\section{Liver
Cancer}

\begin{tabular}{|c|c|}
\hline \multicolumn{2}{|c|}{-358} \\
\hline DOI: 10.1159/000496439 & $\begin{array}{l}\text { (c) } 2019 \text { S. Karger AG, Basel } \\
\text { www.karger.com/lic }\end{array}$ \\
\hline
\end{tabular}

Bouattour et al.: Systemic Treatment for Advanced HCC

(9.2 vs. 7.6 months, $p=0.14$ ) [19]. However, a post hoc analysis suggested that patients with AFP levels $\geq 400 \mathrm{ng} / \mathrm{mL}$ could benefit from ramucirumab. A new phase $3 \mathrm{RCT}$ is now comparing ramucirumab with placebo in this subgroup and presentation at the ASCO Annual Meeting reported an increased OS in the ramucirumab arm compared to the placebo arm (NCT02435433) [20]. Apatinib is a TKI, mainly directed against the VEGF receptor, that is currently tested in a phase 3, placebo-controlled RCT in Asia (NCT02329860).

\section{What Toxicity Profiles Can Be Expected When Using Targeted Therapy or}

Immunotherapy?

The currently available therapies have different toxicity profiles and specific AEs can arise at different stages, even if most of AEs of tyrosine kinase inhibitors occur in the first month of treatment. During the first month of treatment with targeted therapies (sorafenib, regorafenib, lenvatinib), arterial hypertension, diarrhea, asthenia, and HFSR may occur. HFSR can emerge several months after the beginning of treatment, but interestingly, retrospective data indicate that early skin toxicity and diarrhea may predict clinical benefit from sorafenib $[44,45]$. After the first month, rash and dry skin are more frequent. Later in the course of treatment, more rare AEs may be seen, such as cardiovascular events and thrombosis. Immunotherapy has a completely different safety profile, mainly consisting of rash and rare, but sometimes severe, immune-mediated AEs including immune-mediated hypothyroidism, adrenal insufficiency, colitis, hepatitis, and acute renal injury. Figure 1 outlines suggested management options for the main AEs related to sorafenib, lenvatinib, regorafenib, and anti-PD1 inhibitors such as nivolumab and pembrolizumab.

What Is the Role of Driver Mutations and Precision Medicine in the Field of HCC?

Important advances have been made in our understanding of the pathophysiology of HCC and signaling pathways involved in hepatocarcinogenesis, including the identification of driver mutations and key signaling pathways (Fig. 2) [46-50]. Promoter mutations in the telomere maintenance gene telomerase reverse transcriptase (TERT) are the most frequent genetic alteration in HCC, occurring in $40-60 \%$ of patients [49]. Mutations in TP53 (25-40\%), CTNNB1 (11-37\%), chromatin remodeling complex (ARID1A, 4-17\%; ARID2, 3-18\%), and the oxidative stress pathway (NFE2L2, 3-6\%; KEAP1, 2-8\%) are also common in HCC.

Most approved treatments in advanced HCC are TKIs targeting multiple targets. However, phase 3 RCTs did not include biomarker enrichment, except for those on ramucirumab and tivantinib. The concept of precision medicine has been built on our knowledge about the cancer genome. Biomarkers derived from tumor analysis have been suggested as potential predictors of response to sorafenib, such as VEGFA amplification and FGF3/4 amplification. However, these biomarkers have only been analyzed in retrospective studies and lack prospective validation [51]. Overall, compared to other types of solid tumors, precision medicine in HCC is still at the embryonical stage.

Another example of precision medicine in HCC is the use of c-Met inhibitors in patients harboring high expression of MET [52]. Unfortunately, nonselective kinase inhibitors with c-Met activity, such as tivantinib, have not shown efficacy in advanced HCC [31]. This might be due to incomplete understanding of the mechanism of action of the drug (preclinical data has recently suggested that tivantinib is not a potent Met inhibitor after all) and the limited value of MET overexpression as a surrogate marker of oncogene addiction [53]. Another example is the FGFR4 inhibitor BLU-554. Preliminary phase 1 data with BLU-554 in patients with FGF19 overexpression show limited toxicity and promising clinical activity [54].

Precision medicine in HCC has several limitations. Potential targetable alterations (e.g., FGF19 amplifications, PTEN deletion, and RPS6KA3 inactivation) have a frequency of less than $5-10 \%$ in the HCC population [55]. The most frequent genetic alterations in HCC currently 


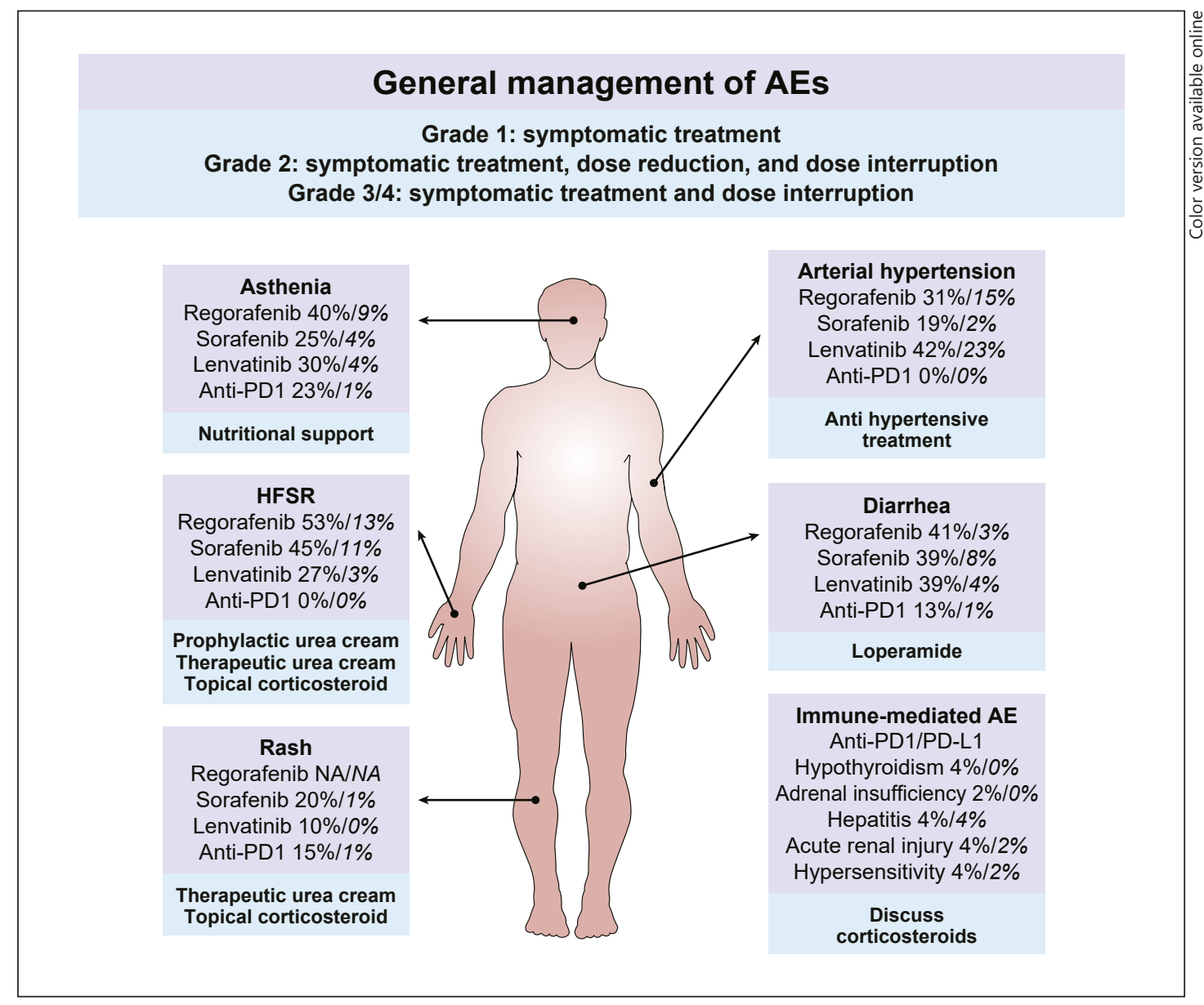

Fig. 1. Managements of AEs of systemic treatments for HCC. The purple boxes contain the main AEs related to sorafenib, lenvatinib, regorafenib, and anti-PD1 antibodies, with the percentages of any grade AEs/grade 3-4 AEs (italics). The blue boxes contain suggestions for the management of the AEs. AE, adverse event; NA, not applicable; HFSR, hand-foot-skin reaction; PD1, programmed cell death protein 1; PD-L1, programmed cell death protein ligand.

have no available treatment options and tumor heterogeneity could decrease the efficacy of targeted therapy [56]. Moreover, primary or secondary resistance invariably occurred [57]. Additional knowledge about tumor heterogeneity and plasticity of cancer cells and further efforts on drug development will be required to bypass these drawbacks and lead HCC into the era of precision medicine. In the design of new clinical trial in HCC, biomarker enrichment and tumor biopsies should be mandatory prior to inclusion in order to give access to material that can be used to identify biomarkers of response.

\section{What Are the Consequences of Recent Developments for the Design of Future Clinical}

Trials?

In future phase 3 RCTs, sorafenib or lenvatinib should be the first-line comparators. However, availability of several second-line treatments could lead to an increased postprogression survival after first-line treatment, leading to more difficulties to assess OS in the first line [58]. Regorafenib and cabozantinib should be second-line comparators in patients who previously tolerated sorafenib. Once approved, cabozantinib should be the comparator in 


\section{Liver
Cancer}

\begin{tabular}{|c|c|}
\hline \multicolumn{2}{|c|}{-358} \\
\hline DOI: 10.1159/000496439 & $\begin{array}{l}\text { (c) } 2019 \text { S. Karger AG, Basel } \\
\text { www.karger.com/lic }\end{array}$ \\
\hline
\end{tabular}

Bouattour et al.: Systemic Treatment for Advanced HCC

second-line treatment of sorafenib-intolerant patients (as defined in the RESORCE trial: sorafenib $\geq 400 \mathrm{mg} /$ day for $\geq 20$ of the last 28 days of treatment). Placebo-controlled trials can be acceptable in the third line. Further data from phase 3 RCTs with nivolumab and pembrolizumab in the first and second line are needed to determine their place in future RCTs.

Controversies exist about the use of surrogate endpoints, including TTP, PFS, or ORR, in patients with advanced HCC. ORR by the mRECIST has been suggested as a validated surrogate endpoint, but this remains controversial $[59,60]$. Phase 3 studies have shown discrepancies between OS and proposed surrogate endpoints, including increases in ORR, PFS or TTP without an increased OS (Table 1). This could be explained by a conceivably higher toxicity of the novel agents or increased tumor aggressiveness after progression [61]. Currently, OS remains the primary endpoint in phase 3 RCTs in advanced HCC.

\section{Patient Scenarios and Treatment Proposals}

For a decade, sorafenib was the only approved treatment for advanced HCC, with no other approved options for sorafenib-intolerant and/or progressive patients. Positive results of novel compounds in first, second and even further lines of treatment prompt physicians to question the position of new treatment options and develop tailored treatment strategies, taking into account clinical characteristics, underlying disease, HCC stage, and reimbursement status. However, clinicians have to interpret the results of subgroup analysis from RCTs with caution. This section contains suggested scenarios, treatment proposals, and sequences to aid the physician's decision-making process around the treatment of advanced HCC (Fig. 3). We discuss these scenarios as clinicians could discuss treatment options in different clinical situations in a multidisciplinary tumor board. However, most data come from subgroup analyses that cannot provide definitive proof for each scenario.

\section{First-Line Treatment}

HCV-Infected Patients with Advanced HCC

Sorafenib has shown a clear efficacy and safety benefit in HCV-infected patients with advanced HCC [17]. A subgroup analysis of the SHARP and AP trials showed that HCV-positive patients were more likely to benefit from sorafenib [15]. Sorafenib could be suggested as a first-line option in this population.

Nivolumab showed impressive efficacy results in a phase 1/2 study in the second line, but adequate data is lacking to recommend nivolumab as a first-line treatment option [9]. Final results of the phase 3 head-to-head RCT comparing nivolumab and sorafenib can shed light on possible advantages in survival and cost-effectiveness.

HBV-Infected Patients with Advanced HCC

Sorafenib has shown a clear benefit compared with placebo in HBV-infected patients with advanced HCC $[6,15]$. However, a recent meta-analysis of three RCTs with sorafenib $(n=1,643)$ indicated lower benefit of sorafenib in this population [17]. Despite the contravened conclusion of this study, this finding needs to be considered when treating HBV-infected patients [62].

Phase 3 data indicate that lenvatinib may have more benefit than sorafenib in HBVinfected patients, but this assumption is only based on a subgroup analysis of the phase 3 RCT [18]. Lenvatinib seemed to have a manageable toxicity profile. Furthermore, costs are 


\section{Liver \\ Cancer}

\begin{tabular}{|c|c|}
\hline \multicolumn{2}{|l|}{ Li } \\
\hline DOI: $10.1159 / 000496439$ & $\begin{array}{l}\text { (c) } 2019 \text { S. Karger AG, Basel } \\
\text { www.karger.com/lic }\end{array}$ \\
\hline
\end{tabular}

Bouattour et al.: Systemic Treatment for Advanced HCC

currently lower than that of sorafenib, which is relevant considering HBV infections are more frequent in developing countries.

The subgroup analysis of the REFLECT trial could permit starting with first-line lenvatinib (where approved) in HBV-positive patients with advanced HCC to optimize their chance of

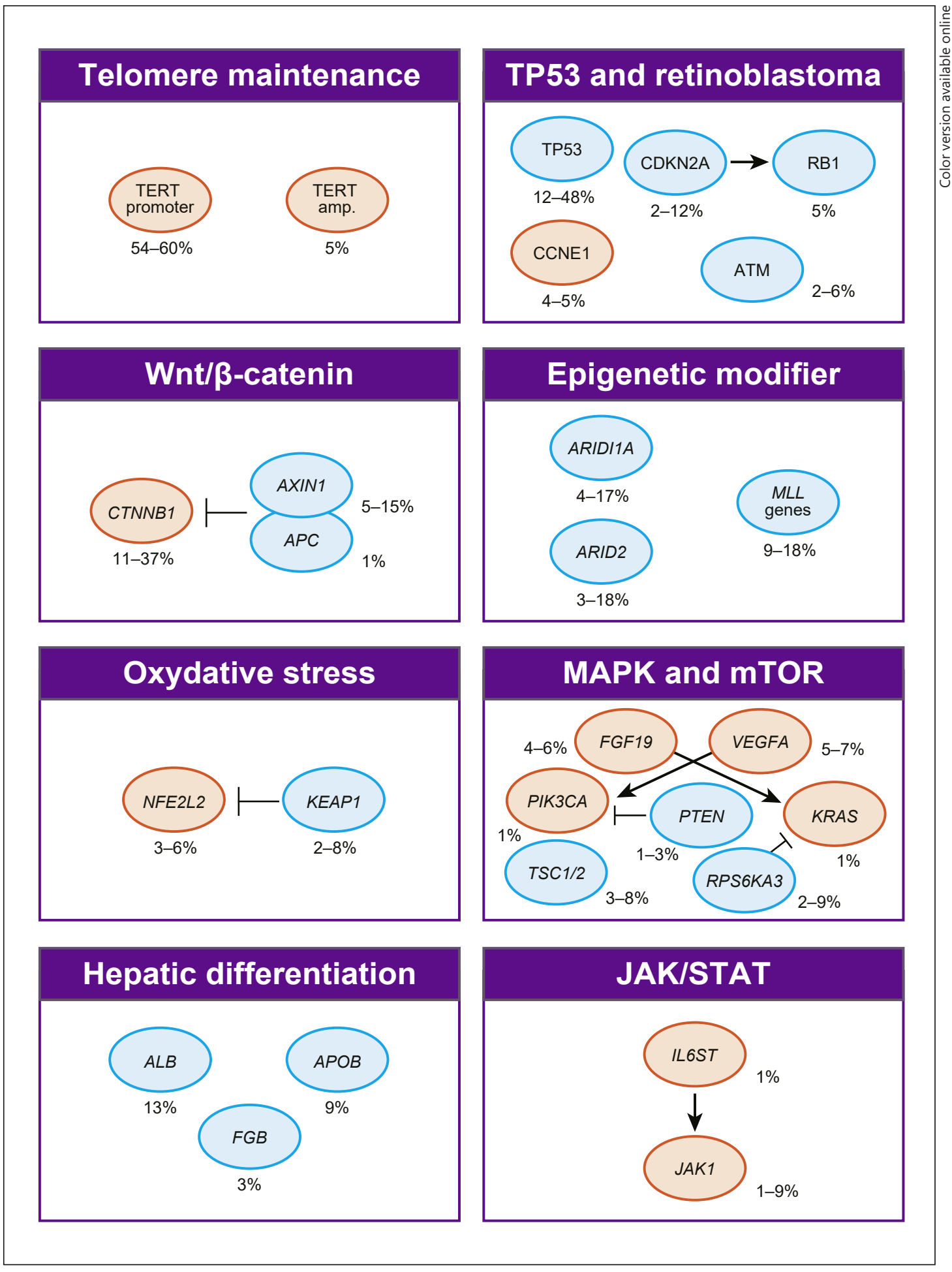

(For legend see next page.) 


\section{Liver Cancer}

Fig. 3. Current and future treatment options by line of therapy and patient subgroup. The green boxes contain recommended (future) therapies. The orange boxes contain alternative future treatments, not recommended as first choice based on the current knowledge. No studies on secondline treatment after progression/ intolerance to lenvatinib are currently available. * Insufficient data for robust recommendation. † Only registered in the US. ${ }^{\ddagger}$ Not registered. BCLC, Barcelona Clinic Liver Cancer; ECOG-PS, Eastern Cooperative Oncology Group performance status; pts, patients; RCT, randomized controlled trial.

\begin{tabular}{|c|c|}
\hline \multicolumn{2}{|c|}{ Liver Cancer 2019;8:341-358 } \\
\hline DOI: 10.1159/000496439 & $\begin{array}{l}\text { (c) } 2019 \text { S. Karger AG, Basel } \\
\text { www.karger.com/lic }\end{array}$ \\
\hline
\end{tabular}

Bouattour et al.: Systemic Treatment for Advanced HCC

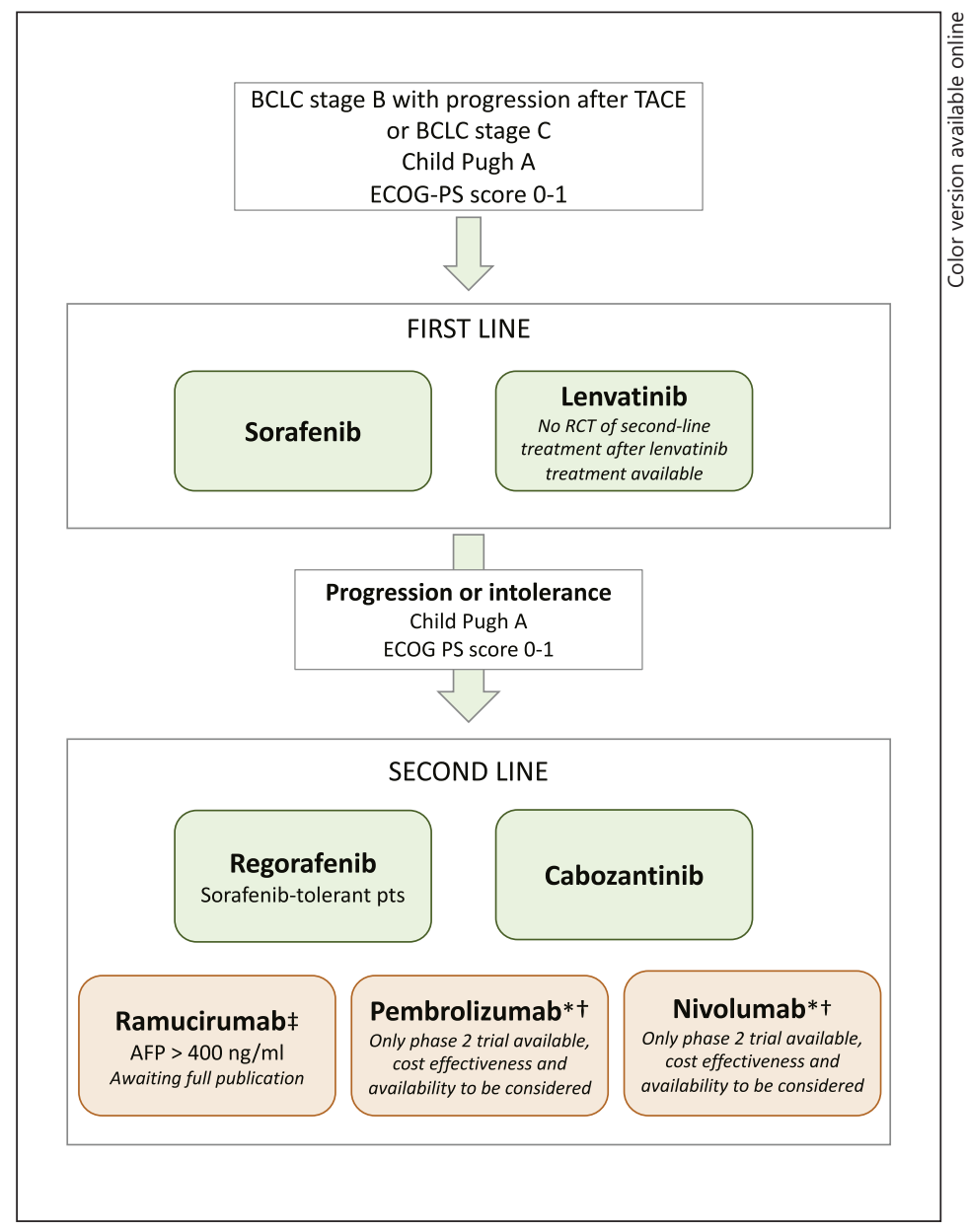

Fig. 2. Mutational landscape of HCC. This figure depicts the main signaling pathways mutated in liver carcinogenesis. The main driver genes mutated in HCC in each signaling pathway are shown with the percentages of somatic mutations in each gene. Oncogenes are presented in red and tumor suppressor genes in blue. TERT, CCNE1, and MLL4 are also targeted by recurrent somatic HBV insertions. The TERT gene was either mutated in its promoter or amplified (TERT amp.). ALB, albumin; APC, adenomatosis polyposis coli tumor suppressor; APOB, apolipoprotein B; ARID2, AT-rich interactive domain-containing protein 2; ARIDI1A, ATrich interactive domain-containing protein $1 \mathrm{~A}$; ATM, ataxia-telangiectasia mutated; CCNE1, cyclin E1; CDKN2A, cyclin-dependent kinase inhibitor 2A; CTNNB1, Catenin (cadherin-associated protein) beta 1; FBG, fibrinogen beta chain; FGF19, fibroblast growth factor 19; IL6ST, glycoprotein 130; JAK, janus kinase; KEAP1, kelch-like ECH-associated protein 1; MLL, mixed-lineage leukemia; mTOR, mechanistic target of rapamycin; NFE2 L2, nuclear factor (erythroid-derived 2)-like 2; RB1, retinoblastoma protein; PIK2CA, phosphatidylinositol-4,5-bisphosphate 3-kinase, catalytic subunit alpha; PTEN, phosphatase and tensin homolog; RPS6KA3, ribosomal protein S6 kinase, 90kDa, polypeptide 3; STAT, signal transducer and activator of transcription; TERT, telomerase reverse transcriptase; TP53, tumor protein p53; TSC1/2, tuberous sclerosis 1/2; VEGFA, vascular endothelial growth factor A. 


\section{Liver Cancer}

\begin{tabular}{|c|c|}
\hline \multicolumn{2}{|l|}{ Liver Cancer 2019;8:341-358 } \\
\hline DOI: 10.1159/000496439 & $\begin{array}{l}\text { (c) } 2019 \text { S. Karger AG, Basel } \\
\text { www.karger.com/lic }\end{array}$ \\
\hline
\end{tabular}

Bouattour et al.: Systemic Treatment for Advanced HCC

response, survival, and treatment cost. It is also possible to treat HBV-positive patients with sorafenib, as the AP trial included a large proportion of HBV-infected patients and showed benefit. Sorafenib could be a treatment option for patients with comorbidities, especially arterial hypertension, bearing in mind the relatively high incidence of this disease as a lenvatinib-related AE. While awaiting the final phase 3 data, nivolumab will be not suggested for first-line use.

Patients with Locally Advanced HCC

The subgroup of patients with locally advanced HCC (BCLC stage C with portal vein invasion, without metastases or BCLC stage B progressive after TACE) was the target of two open-label phase 3 RCTs evaluating sorafenib versus selective internal radiation therapy (SIRT) (SARAH and SIRVENIB) [63, 64]. Despite a lower rate of AEs, a better quality of life, and higher ORR, survival benefit was not significantly higher in the SIRT groups and OS was slightly lower in the SIRT group compared with the sorafenib arm in patients with portal vein thrombosis. Sorafenib therefore remains the standard of care in this setting.

\section{Second and Further Lines of Treatment}

Patients with Progression under Sorafenib

For many years, patients who progressed under sorafenib had no other options than to continue sorafenib, receive best supportive care, or participate in (few) clinical trials. Nowadays, it is estimated that around $50 \%$ of patients are candidates for second-line treatment [65].

Regorafenib has shown a clear benefit in progressive patients, decreasing the risk of death by $37 \%$ versus best supportive care, provided sorafenib was well tolerated (sorafenib $\geq 400 \mathrm{mg}$ /day for $\geq 20$ of the last 28 days of treatment in the RESORCE trial) [8]. However, the number of treatment options is increasing, as exemplified by the FDA approval of nivolumab and pembrolizumab. In our opinion, regorafenib and, after approval and publication of the full data, cabozantinib should be the second-line treatment options of choice, pending the final survival data from nivolumab and pembrolizumab (expected in 2019) and the publication on ramucirumab in patients with AFP $\geq 400 \mathrm{ng} / \mathrm{mL}$. The efficacy of nivolumab and pembrolizumab should be balanced by the costs, patient preference (oral vs. intravenous administration), and reimbursement.

A small proportion of patients will be eligible for third-line treatment. Cabozantinib may be a future treatment option in this subgroup. Finally, no results of phase 3 trials are available for second-line treatment after lenvatinib, resulting in the absence of a robust recommendation in this setting.

Sorafenib-Intolerant Patients

When no second-line therapies were available, intolerant patients continued sorafenib with adapted doses. Currently, nivolumab and pembrolizumab could be considered in the US. Cabozantinib and ramucirumab (in patients with AFP $\geq 400 \mathrm{ng} / \mathrm{mL}$ ) may present future alternatives but are not yet approved. Regorafenib should generally be avoided in sorafenib-intolerant patients, but if no alternative options are available, regorafenib could be administered with caution, on a case-by-case basis, depending on the toxicities patients experienced with sorafenib. Close monitoring would be essential, as these patients were excluded from the RESORCE trial.

\section{Conclusion}

After 10 years, sorafenib is still the first-line standard of care for many patients with advanced HCC, including patients with locally advanced HCC and HCV-related advanced HCC. Lenvatinib may present an alternative for patients. Regorafenib is the treatment of choice for progressive patients, although nivolumab and pembrolizumab is available in the US based on 
Bouattour et al.: Systemic Treatment for Advanced HCC

the results of a phase 2 study. Ramucirumab (in patients with AFP $\geq 400 \mathrm{ng} / \mathrm{mL}$ ) and cabozantinib (in an unselected population) may present future second-line treatment options. In the near future, more data will be published on immunotherapy that will possibly affect the herein proposed recommendations. In the more distant future, precision medicine may come into play in the treatment of advanced HCC.

\section{Acknowledgements}

This review article was written on behalf of HCC CONNECT; for more information, visit www.hccconnect.info. HCC CONNECT is supported by an Independent Educational Grant from Bayer. Bayer did not have a critical role in the design and conduct of the study; collection, management, analysis, and interpretation of the data; preparation, review and approval of the manuscript; or decision to submit the manuscript for publication. Editorial support was provided by Kim Grootscholten, MSc of COR2ED.

\section{Disclosure Statement}

M.B. is a consultant for and has received honoraria from Bayer Pharma, Bristol-Myers Squibb, Sitex Medical. A.R.H. is a consultant for and has received honoraria from Bayer Pharma, Bristol-Myers Squibb, Merck. N.M., E.I.C., and J.-C.N. have declared no potential conflicts of interest.

\section{References}

1 Ervik M, Lam F, Ferlay J, et al. Cancer Today. Lyon, France: International Agency for Research on Cancer; 2016 [cited 2018 Feb 23]. Available from: http://gco.iarc.fr/today.

2 Galle PR, Forner A, Llovet JM, Mazzaferro V, Piscaglia F, Raoul JL, et al.; European Association for the Study of the Liver. Electronic address: easloffice@easloffice.eu; European Association for the Study of the Liver. EASL clinical practice guidelines: management of hepatocellular carcinoma. J Hepatol. 2018 Jul;69(1):182-236.

3 Forner A, Reig M, Bruix J. Hepatocellular carcinoma. Lancet. 2018 Mar;391(10127):1301-14.

4 Llovet JM, Ricci S, Mazzaferro V, Hilgard P, Gane E, Blanc JF, et al.; SHARP Investigators Study Group. Sorafenib in advanced hepatocellular carcinoma. N Engl J Med. 2008 Jul;359(4):378-90.

5 Bruix J, Raoul JL, Sherman M, Mazzaferro V, Bolondi L, Craxi A, et al. Efficacy and safety of sorafenib in patients with advanced hepatocellular carcinoma: subanalyses of a phase III trial. J Hepatol. 2012 Oct;57(4):821-9.

6 Cheng AL, Kang YK, Chen Z, Tsao CJ, Qin S, Kim JS, et al. Efficacy and safety of sorafenib in patients in the AsiaPacific region with advanced hepatocellular carcinoma: a phase III randomised, double-blind, placebocontrolled trial. Lancet Oncol. 2009 Jan;10(1):25-34.

7 Heimbach JK, Kulik LM, Finn RS, Sirlin CB, Abecassis MM, Roberts LR, et al. AASLD guidelines for the treatment of hepatocellular carcinoma. Hepatology. 2018 Jan;67(1):358-80.

8 Bruix J, Qin S, Merle P, Granito A, Huang YH, Bodoky G, et al.; RESORCE Investigators. Regorafenib for patients with hepatocellular carcinoma who progressed on sorafenib treatment (RESORCE): a randomised, doubleblind, placebo-controlled, phase 3 trial. Lancet. 2017 Jan;389(10064):56-66.

9 El-Khoueiry AB, Sangro B, Yau T, Crocenzi TS, Kudo M, Hsu C, et al. Nivolumab in patients with advanced hepatocellular carcinoma (CheckMate 040): an open-label, non-comparative, phase $1 / 2$ dose escalation and expansion trial. Lancet. 2017 Jun;389(10088):2492-502.

10 Pinter M, Sieghart W, Graziadei I, Vogel W, Maieron A, Königsberg R, et al. Sorafenib in unresectable hepatocellular carcinoma from mild to advanced stage liver cirrhosis. Oncologist. 2009 Jan;14(1):70-6.

11 Ozenne V, Paradis V, Pernot S, Castelnau C, Vullierme MP, Bouattour M, et al. Tolerance and outcome of patients with unresectable hepatocellular carcinoma treated with sorafenib. Eur J Gastroenterol Hepatol. 2010 Sep; 22(9):1106-10.

12 Abou-Alfa GK, Schwartz L, Ricci S, Amadori D, Santoro A, Figer A, et al. Phase II study of sorafenib in patients with advanced hepatocellular carcinoma. J Clin Oncol. 2006 Sep;24(26):4293-300.

13 Wörns MA, Koch S, Niederle IM, Marquardt JU, Nguyen-Tat M, Gamstätter T, et al. The impact of patient and tumour baseline characteristics on the overall survival of patients with advanced hepatocellular carcinoma treated with sorafenib. Dig Liver Dis. 2013 May;45(5):408-13.

14 Marrero JA, Kudo M, Venook AP, Ye SL, Bronowicki JP, Chen XP, et al. Observational registry of sorafenib use in clinical practice across Child-Pugh subgroups: the GIDEON study. J Hepatol. 2016 Dec;65(6):1140-7. 
15 Bruix J, Cheng AL, Meinhardt G, Nakajima K, De Sanctis Y, Llovet J. Prognostic factors and predictors of sorafenib benefit in patients with hepatocellular carcinoma: analysis of two phase III studies. J Hepatol. 2017 Nov;67(5):999-1008.

16 Cantarini MC, Trevisani F, Morselli-Labate AM, Rapaccini G, Farinati F, Del Poggio P, et al.; Italian Liver Cancer (ITA.LI.CA) group. Effect of the etiology of viral cirrhosis on the survival of patients with hepatocellular carcinoma. Am J Gastroenterol. 2006 Jan;101(1):91-8.

17 Jackson R, Psarelli EE, Berhane S, Khan H, Johnson P. Impact of Viral Status on Survival in Patients Receiving Sorafenib for Advanced Hepatocellular Cancer: A Meta-Analysis of Randomized Phase III Trials. J Clin Oncol. $2017 \mathrm{Feb} ; 35(6): 622-8$.

18 Kudo M, Finn RS, Qin S, Han KH, Ikeda K, Piscaglia F, et al. Lenvatinib versus sorafenib in first-line treatment of patients with unresectable hepatocellular carcinoma: a randomised phase 3 non-inferiority trial. Lancet. 2018 Mar;391(10126):1163-73.

19 Zhu AX, Park JO, Ryoo BY, Yen CJ, Poon R, Pastorelli D, et al.; REACH Trial Investigators. Ramucirumab versus placebo as second-line treatment in patients with advanced hepatocellular carcinoma following first-line therapy with sorafenib (REACH): a randomised, double-blind, multicentre, phase 3 trial. Lancet Oncol. 2015 Jul;16(7):859-70.

20 Zhu AX, Kang YK, Yen CJ, Finn RS, Galle PR, Llovet JM, et al. REACH-2: A randomized, double-blind, placebocontrolled phase 3 study of ramucirumab versus placebo as second-line treatment in patients with advanced hepatocellular carcinoma (HCC) and elevated baseline alpha-fetoprotein (AFP) following first-line sorafenib [abstract]. J Clin Oncol. 2018;36(15_suppl Suppl):4003.

21 Rimassa L, Pressiani T, Boni C, Carnaghi C, Rota Caremoli E, Fagiuoli S, et al. A phase II randomized dose escalation trial of sorafenib in patients with advanced hepatocellular carcinoma. Oncologist. 2013;18(4):379-80.

22 Qin S, Bai Y, Lim HY, Thongprasert S, Chao Y, Fan J, et al. Randomized, multicenter, open-label study of oxaliplatin plus fluorouracil/leucovorin versus doxorubicin as palliative chemotherapy in patients with advanced hepatocellular carcinoma from Asia. J Clin Oncol. 2013 Oct;31(28):3501-8.

23 Cheng AL, Kang YK, Lin DY, Park JW, Kudo M, Qin S, et al. Sunitinib versus sorafenib in advanced hepatocellular cancer: results of a randomized phase III trial. J Clin Oncol. 2013 Nov;31(32):4067-75.

24 Johnson PJ, Qin S, Park JW, Poon RT, Raoul JL, Philip PA, et al. Brivanib versus sorafenib as first-line therapy in patients with unresectable, advanced hepatocellular carcinoma: results from the randomized phase III BRISK-FL study. J Clin Oncol. 2013 Oct;31(28):3517-24.

25 Zhu AX, Rosmorduc O, Evans TR, Ross PJ, Santoro A, Carrilho FJ, et al. SEARCH: a phase III, randomized, doubleblind, placebo-controlled trial of sorafenib plus erlotinib in patients with advanced hepatocellular carcinoma. J Clin Oncol. 2015 Feb;33(6):559-66.

26 Cainap C, Qin S, Huang WT, Chung IJ, Pan H, Cheng Y, et al. Linifanib versus Sorafenib in patients with advanced hepatocellular carcinoma: results of a randomized phase III trial. J Clin Oncol. 2015 Jan;33(2):172-9.

27 Llovet JM, Decaens T, Raoul JL, Boucher E, Kudo M, Chang C, et al. Brivanib in patients with advanced hepatocellular carcinoma who were intolerant to sorafenib or for whom sorafenib failed: results from the randomized phase III BRISK-PS study. J Clin Oncol. 2013 Oct;31(28):3509-16.

28 Zhu AX, Kudo M, Assenat E, Cattan S, Kang YK, Lim HY, et al. Effect of everolimus on survival in advanced hepatocellular carcinoma after failure of sorafenib: the EVOLVE-1 randomized clinical trial. JAMA. 2014 Jul;312(1): 57-67.

29 Abou-Alfa GK, Niedzwieski D, Knox JJ, Kaubisch A, Posey J, Tan BR, et al. Phase III randomized study of sorafenib plus doxorubicin versus sorafenib in patients with advanced hepatocellular carcinoma (HCC): CALGB 80802 (Alliance) [abstract]. J Clin Oncol. 2016;34(15_suppl Suppl):4003.

30 Kudo M, Moriguchi M, Numata K, Hidaka H, Tanaka H, Ikeda M, et al. S-1 versus placebo in patients with sorafenib-refractory advanced hepatocellular carcinoma (S-CUBE): a randomised, double-blind, multicentre, phase 3 trial. Lancet Gastroenterol Hepatol. 2017 Jun;2(6):407-17.

31 Rimassa L, Assenat E, Peck-Radosavljevic M, Pracht M, Zagonel V, Mathurin P, et al. Tivantinib for second-line treatment of MET-high, advanced hepatocellular carcinoma (METIV-HCC): a final analysis of a phase 3, randomised, placebo-controlled study. Lancet Oncol. 2018 May;19(5):682-93.

32 Abou-Alfa GK, Qin S, Ryoo BY, Lu SN, Yen CJ, Feng YH, et al. Phase III randomized study of second line ADI-PEG 20 plus best supportive care versus placebo plus best supportive care in patients with advanced hepatocellular carcinoma. Ann Oncol. 2018 Jun;29(6):1402-8.

33 Nault JC. The end of almost 10 years of negative RCTs in advanced hepatocellular carcinoma. Lancet. 2017 Jan; 389(10064):4-6.

34 Finn RS, Merle P, Granito A, Huang YH, Bodoky G, Pracht M, et al. Outcomes of sequential treatment with sorafenib followed by regorafenib for HCC: additional analyses from the phase III RESORCE trial. J Hepatol. 2018 Aug;69(2):353-8.

35 Gyawali B, Prasad V. Health policy: me-too drugs with limited benefits - the tale of regorafenib for HCC. Nat Rev Clin Oncol. 2017 Nov;14(11):653-4.

36 Zhu AX, Finn RS, Edeline J, Cattan S, Ogasawara S, Palmer D, et al.; KEYNOTE-224 investigators. Pembrolizumab in patients with advanced hepatocellular carcinoma previously treated with sorafenib (KEYNOTE224): a non-randomised, open-label phase 2 trial. Lancet Oncol. 2018 Jul;19(7):940-52.

37 Ikeda K, Kudo M, Kawazoe S, Osaki Y, Ikeda M, Okusaka T, et al. Phase 2 study of lenvatinib in patients with advanced hepatocellular carcinoma. J Gastroenterol. 2017 Apr;52(4):512-9. 
Bouattour et al.: Systemic Treatment for Advanced HCC

38 Kelley RK, Verslype C, Cohn AL, Yang TS, Su WC, Burris H, et al. Cabozantinib in hepatocellular carcinoma: results of a phase 2 placebo-controlled randomized discontinuation study. Ann Oncol. 2017 Mar;28(3): 528-34.

39 Abou-Alfa GK, Meyer T, Cheng AL, El-Khoueiry AB, Rimassa L, Ryoo BY, et al. Cabozantinib in Patients with Advanced and Progressing Hepatocellular Carcinoma. N Engl J Med. 2018 Jul;379(1):54-63.

40 Sangro B, Gomez-Martin C, de la Mata M, Iñarrairaegui M, Garralda E, Barrera P, et al. A clinical trial of CTLA-4 blockade with tremelimumab in patients with hepatocellular carcinoma and chronic hepatitis C. J Hepatol. 2013 Jul;59(1):81-8.

41 Stein S, Pishvaian MJ, Lee MS, Lee KH, Hernandez S, Kwan A, et al. Safety and clinical activity of 1L atezolizumab + bevacizumab in a phase Ib study in hepatocellular carcinoma (HCC) [abstract]. J Clin Oncol. 2018;36(15_ suppl Suppl):4074.

42 Finn RS, Ducreux M, Qin S, Galle PR, Zhu AX, Ikeda M, et al. IMbrave150: A randomized phase III study of 1L atezolizumab plus bevacizumab vs sorafenib in locally advanced or metastatic hepatocellular carcinoma [abstract]. J Clin Oncol. 2018;36(15_suppl Suppl):TPS4141.

43 Ikeda M, Sung MX, Kudo M, Kobayashi M, Baron AD, Finn RS, et al. A phase $1 \mathrm{~b}$ trial of lenvatinib (LEN) plus pembrolizumab (PEM) in patients (pts) with unresectable hepatocellular carcinoma (uHCC) [abstract]. J Clin Oncol. 2018;36(15_suppl Suppl):4076.

44 Cho JY, Paik YH, Lim HY, Kim YG, Lim HK, Min YW, et al. Clinical parameters predictive of outcomes in sorafenibtreated patients with advanced hepatocellular carcinoma. Liver Int. 2013 Jul;33(6):950-7.

45 Reig M, Torres F, Rodriguez-Lope C, Forner A, LLarch N, Rimola J, et al. Early dermatologic adverse events predict better outcome in HCC patients treated with sorafenib. J Hepatol. 2014 Aug;61(2):318-24.

46 Schulze K, Imbeaud S, Letouzé E, Alexandrov LB, Calderaro J, Rebouissou S, et al. Exome sequencing of hepatocellular carcinomas identifies new mutational signatures and potential therapeutic targets. Nat Genet. 2015 May;47(5):505-11.

47 Totoki Y, Tatsuno K, Covington KR, Ueda H, Creighton CJ, Kato M, et al. Trans-ancestry mutational landscape of hepatocellular carcinoma genomes. Nat Genet. 2014 Dec;46(12):1267-73.

48 Guichard C, Amaddeo G, Imbeaud S, Ladeiro Y, Pelletier L, Maad IB, et al. Integrated analysis of somatic mutations and focal copy-number changes identifies key genes and pathways in hepatocellular carcinoma. Nat Genet. 2012 May;44(6):694-8.

49 Nault JC, Mallet M, Pilati C, Calderaro J, Bioulac-Sage P, Laurent C, et al. High frequency of telomerase reversetranscriptase promoter somatic mutations in hepatocellular carcinoma and preneoplastic lesions. Nat Commun. 2013;4(1):2218.

50 Sawey ET, Chanrion M, Cai C, Wu G, Zhang J, Zender L, et al. Identification of a therapeutic strategy targeting amplified FGF19 in liver cancer by Oncogenomic screening. Cancer Cell. 2011 Mar;19(3):347-58.

51 Horwitz E, Stein I, Andreozzi M, Nemeth J, Shoham A, Pappo O, et al. Human and mouse VEGFA-amplified hepatocellular carcinomas are highly sensitive to sorafenib treatment. Cancer Discov. 2014 Jun;4(6): 730-43.

52 Bouattour M, Raymond E, Qin S, Cheng AL, Stammberger U, Locatelli G, et al. Recent developments of c-Met as a therapeutic target in hepatocellular carcinoma. Hepatology. 2018 Mar;67(3):1132-49.

53 Rebouissou S, La Bella T, Rekik S, Imbeaud S, Calatayud AL, Rohr-Udilova N, et al. Proliferation Markers Are Associated with MET Expression in Hepatocellular Carcinoma and Predict Tivantinib Sensitivity In Vitro. Clin Cancer Res. 2017 Aug;23(15):4364-75.

54 Kang YK, Macarulla T, Yau T, et al. Clinical activity of BLU-554, a potent, highly-selective fgfr4 inhibitor in advanced hepatocellular carcinoma (HCC) with FGFR4 pathway activation [abstract]. 11th ILCA Annual Conference. Seoul; 2017. p. 0-032.

55 Schulze K, Nault JC, Villanueva A. Genetic profiling of hepatocellular carcinoma using next-generation sequencing. J Hepatol. 2016 Nov;65(5):1031-42.

56 Le Tourneau C, Delord JP, Gonçalves A, Gavoille C, Dubot C, Isambert N, et al.; SHIVA investigators. Molecularly targeted therapy based on tumour molecular profiling versus conventional therapy for advanced cancer (SHIVA): a multicentre, open-label, proof-of-concept, randomised, controlled phase 2 trial. Lancet Oncol. 2015 Oct;16(13):1324-34.

57 Prasad V, Fojo T, Brada M. Precision oncology: origins, optimism, and potential. Lancet Oncol. 2016 Feb; 17(2):e81-6.

58 Broglio KR, Berry DA. Detecting an overall survival benefit that is derived from progression-free survival. J Natl Cancer Inst. 2009 Dec;101(23):1642-9.

59 Lencioni R, Llovet JM. Modified RECIST (mRECIST) assessment for hepatocellular carcinoma. Semin Liver Dis. 2010 Feb;30(1):52-60.

60 Lencioni R, Montal R, Torres F, Park JW, Decaens T, Raoul JL, et al. Objective response by mRECIST as a predictor and potential surrogate end-point of overall survival in advanced HCC. J Hepatol. 2017 Jun;66(6): 1166-72.

61 Llovet JM, Hernandez-Gea V. Hepatocellular carcinoma: reasons for phase III failure and novel perspectives on trial design. Clin Cancer Res. 2014 Apr;20(8):2072-9.

62 Personeni N, Rimassa L, Giordano L, Santoro A. Sorafenib in Hepatitis C Virus-Negative Patients With Hepatocellular Carcinoma: Don't Throw the Baby Out With the Bathwater! J Clin Oncol. 2017 Jul;35(19): 2213-4. 
63 Vilgrain V, Pereira H, Assenat E, Guiu B, Ilonca AD, Pageaux GP, et al.; SARAH Trial Group. Efficacy and safety of selective internal radiotherapy with yttrium-90 resin microspheres compared with sorafenib in locally advanced and inoperable hepatocellular carcinoma (SARAH): an open-label randomised controlled phase 3 trial. Lancet Oncol. 2017 Dec;18(12):1624-36.

64 Chow PK, Gandhi M, Tan SB, Khin MW, Khasbazar A, Ong J, et al.; Asia-Pacific Hepatocellular Carcinoma Trials Group. SIRveNIB: Selective Internal Radiation Therapy Versus Sorafenib in Asia-Pacific Patients With Hepatocellular Carcinoma. J Clin Oncol. 2018 Jul;36(19):1913-21.

65 Iavarone M, Cabibbo G, Biolato M, Della Corte C, Maida M, Barbara M, et al. Predictors of survival in patients with advanced hepatocellular carcinoma who permanently discontinued sorafenib. Hepatology. 2015 Sep; 62(3):784-91. 